\title{
miR-23a-3p/SIX1 regulates glucose uptake and proliferation through GLUT3 in head and neck squamous cell carcinomas
}

\author{
Hongming Wang ${ }^{1 *}$, Weishuang Xue ${ }^{2^{*}}$, Wunyu Ouyang1, Xiaoze Jiang ${ }^{1}$, Xuejun Jiang ${ }^{1 凶}$ \\ 1. Department of Otolaryngology, The First Affiliated Hospital of China Medical University, Shenyang, Liaoning, China. \\ 2. Department of Neurology, The First Affiliated Hospital of China Medical University, Shenyang, Liaoning, China. \\ ${ }^{*}$ Equal contribution \\ $\bowtie$ Corresponding author: Hongming Wang, Department of Otolaryngology, The First Affiliated Hospital of China Medical University, Shenyang 110001, China. \\ Email: wanghongming@cmu1h.com
}

() The author(s). This is an open access article distributed under the terms of the Creative Commons Attribution License (https://creativecommons.org/licenses/by/4.0/). See http://ivyspring.com/terms for full terms and conditions.

Received: 2018.10.26; Accepted: 2020.01.15; Published: 2020.02.10

\begin{abstract}
SIXI overexpression has been reported in several cancers. However, its involvement in head and neck squamous cell carcinoma (HNSCC) remains unclear. In this study we investigated the clinical significance and biological roles of SIX1 in HNSCC. SIX1 expression was upregulated in HNSCC and correlated with TNM stage and nodal metastasis. Analysis of TCGA dataset demonstrated that high SIXI expression correlated with poor patient prognosis. Overexpression of SIXI in the Fadu cell line upregulated cell proliferation, colony formation, glucose uptake and ATP production. In contrast, SIXI depletion in the Detroit562 cell line downregulated cell proliferation, colony formation, glucose uptake and ATP production. We analyzed a series of genes involved in glucose metabolism and found that SIXI overexpression upregulated GLUT3, an important glucose transporter, at both mRNA and protein levels. Using the TRANSFAC database, we found that SIX1 had potential binding sites on the GLUT3 promoter, which was validated by chromatin immunoprecipitation (ChIP) assays. Next, we focused on miR-23a-3p, which could target SIX1 in HNSCC cells. The miR-23a-3p mimic downregulated SIX1 expression while the miR-23a-3p inhibitor upregulated SIX1 expression. The binding of miR-23a-3p to the 3'-UTR of SIX1 was confirmed using the luciferase reporter assay. Analysis of TCGA dataset showed a negative correlation between the miR-23a-3p and SIX1. Furthermore, the miR-23a-3p mimic inhibited cell proliferation, ATP production and glucose uptake, which could be rescued by transfection with the SIXI plasmid. In summary, our study demonstrated that SIXI facilitated HNSCC cell growth through regulation of GLUT3 and glucose uptake. miR-23a-3p targeted the SIX1/GLUT3 axis and suppressed glucose uptake and proliferation in HNSCC.
\end{abstract}

Key words: SIX1; head and neck squamous cell carcinoma; glucose metabolism; GLUT3; miR-23a-3p

\section{Introduction}

Head and neck squamous cell carcinoma (HNSCC) is the sixth most common cancer worldwide [1]. HNSCCs are lethal malignancies with a five-year survival rate of only $\sim 50 \%$ [2]. Local recurrence and distant metastasis after conventional therapy appear to be major contributing factors for restricted survival of HNSCC patients [3]. Therefore, understanding the molecular mechanisms of HNSCC progression would help to improve diagnosis and development of novel therapies.

The sine oculis homeobox (SIX) is a family of evolutionarily conserved transcription factors which are characterized by a nucleic acid recognition domain and the SIX domain [4]. The SIX1 gene was first identified as a mammalian homolog of the Drosophila sine oculis gene and is highly conserved in numerous invertebrate and vertebrate species [5]. SIX1 is highly expressed and plays critical roles in 
multiple tissues throughout embryogenesis and development [6-9]. In most adult tissue, SIX1 is not highly expressed; however, increased SIX1 expression has been documented in multiple cancers, including breast cancer [10, 11], ovarian cancer [12], Wilm's tumors [13], lung cancer [14], hepatocellular carcinoma [15, 16] and colorectal cancer [17]. SIX1 mediates tumor initiation and metastasis by regulating various activities of cancer cells, including cell differentiation [18], proliferation [19, 20], the epithelial-mesenchymal transition (EMT) process [10, 21], responsiveness to apoptotic stimuli [12] and genome stability [22]. SIX1 overexpression has been reported to indicate poor prognosis of gliomas [23], breast cancers [24] and osteosarcomas [25]. However, the clinical significance and biological roles of SIX1 in HNSCC remain unclear.

MicroRNAs (miRNAs) are small (20-22nt), tissue specific, non-coding RNA molecules which cause mRNA translational inhibition or degradation by binding to complementary target mRNAs [26]. The miRNAs are predicted to target over $50 \%$ of all human protein-coding genes, enabling them to have numerous regulatory roles in many physiological and developmental processes [27]. Global downregulation of miRNA expression is an apparent feature of various human tumors [5, 28], including HNSCC [29]. Evidence has indicated that miRNAs regulate malignant progression of HNSCC $[30,31]$. Considering the critical roles of microRNAs in HNSCC, we speculated that certain miRNAs may affect SIX1 expression in HNSCC.

In the present study, we explored the expression and clinical significance of SIX1 in HNSCC tissues. We performed loss- and gain-of-function assays to investigate the biological effects and possible mechanisms of SIX1 in HNSCCs. We also explored the potential function of miRNA-23a-3p, which could negatively regulate SIX1 in HNSCCs.

\section{Materials and methods}

\section{Patients and samples}

The study protocol was approved by the Institutional Reviewer Board of China Medical University. Samples were collected from patients who were diagnosed with cancer and received surgical operations in the First Affiliated Hospital of China Medical University between January 2012 and December 2017. None of the patients received radiotherapy or chemotherapy before surgical resection. The histological diagnosis and differentiation grade were evaluated from sections stained with hematoxylin and eosin according to the World Health Organization (WHO) classification guidelines.

\section{Immunohistochemistry}

Immunohistochemical staining of the cancer tissue sections was performed on $4 \mu \mathrm{m}$ thick sections of formalin-fixed, paraffin-embedded tumor samples. Tumor samples were dewaxed with xylol, and rehydrated with a graded series of ethanol. Endogenous peroxidase was inhibited using 3\% $\mathrm{H}_{2} \mathrm{O}_{2}$ for $5 \mathrm{~min}$. Antigen retrieval was performed with citrate buffer in a pressure cooker for $15 \mathrm{~min}$. Ready-to-use goat serum was used for blocking nonspecific binding. Slides were incubated with the primary antibody against SIX1 (1:200, Sigma-Aldrich, St. Louis, MO, USA) at $4^{\circ} \mathrm{C}$ overnight. Subsequently, the slides were incubated with biotinylated secondary antibody (ready to use, Elivision plus Polymer HRP; mouse/rabbit IHC Kit, Maixin, Fuzhou, China) at $37^{\circ} \mathrm{C}$ for $2 \mathrm{~h}$. The staining reaction was performed using the DAB plus kit (Maixin). Counterstaining was carried out using hematoxylin, and the sections were dehydrated in alcohol.

The specimens were viewed randomly by two pathologists randomly. Strong nuclear staining was regarded as positive staining. According to a previous publication, we classified staining intensity into 3 grades [32]. The staining intensity was scored as 0 (none), 1 (weak), 2 (moderate/strong). Percentages were scored as $1: 1-25 \%, 2: 26-50 \% 3: 51-75 \%$ and 4 : $76-100 \%$. Intensity and percentage scores were multiplied to give a final score of $0-8$. SIX1 was designated as low expression: score $<4$; or high expression (overexpression): score $\geq 4$.

\section{Cell culture and transfection}

Cancer cell lines were obtained from the Cell Bank of Type Culture Collection of Chinese Academy of Science(Shanghai, China). Cells were cultured in RPMI-1640 medium with $10 \%$ fetal bovine serum (FBS, Gibco, Gaithersburg, MD, USA), $100 \mu \mathrm{g} / \mathrm{mL}$ streptomycin and $10 \mathrm{U} / \mathrm{mL}$ penicillin in a $70 \%$ humidity incubator with the conditions of $5 \% \mathrm{CO}_{2}$ at $37^{\circ} \mathrm{C}$. The empty plasmid (Origene, Austin, TX, USA), plasmid with the SIX1 gene (Origene), non-targeting transfection control siRNA (Ribobio, Guangzhou, China) and SIX1 siRNA (Ribobio) were transfected into cells using Lipofectamine 3000 (Invitrogen, Waltham, MA, USA) according to the manufacturer's instructions. FBS-free medium was replaced by medium with $10 \%$ FBS after transfection for $4 \mathrm{~h}$.

\section{Quantitative RT-PCR}

Total RNA from cells was extracted using TRIzol reagent (Thermo Fisher, San Jose, CA, USA) according to the manufacture's instruction. RNA was quantified using a spectrophotometer (NanoDrop 2000c, Thermo Fisher, USA). cDNAs were synthesized using 
PrimeScript RT Master Mix (TaKaRa, Dalian, China), and the total RNAs were used as the template. RT-PCR was performed using the SYBR Green Master Mix with the ABI 7500 Fast Real-time PCR Instrument. The thermal profiles consisted of the following: $50^{\circ} \mathrm{C}$ for $2 \mathrm{~min}, 95^{\circ} \mathrm{C}$ for $2 \mathrm{~min}, 45$ cycles of $95^{\circ} \mathrm{C}$ for $15 \mathrm{sec}$ and $60^{\circ} \mathrm{C}$ for $40 \mathrm{sec}$. $\beta$-actin was used as the endogenous control and the fold-change of gene amplification was calculated according to the 2- $\Delta \Delta \mathrm{Ct}$ Method.

\section{MTT cell viability assay}

Cells $\left(5 \times 10^{3} /\right.$ well $)$ were plated in 96 -well plates and cultured for 1, 2, 3, 4 and 5 days respectively at $37^{\circ} \mathrm{C}$. $\quad 20 \mu \mathrm{L}$ of $5 \mu \mathrm{mg} / \mathrm{mL}$ MTT (3- $(4$, 5-dimethylthiazol-2-yl)-2, 5-diphenyltetrazolium bromide; Sigma Aldrich, St. Louis, MO, USA) solution was added to each well and incubated for 4 hours at $37^{\circ} \mathrm{C}$. The supernatant was removed from each well, and $100 \mu \mathrm{l}$ of DMSO was added to dissolve the formazan crystals. The absorbance was measured at $490 \mathrm{~nm}$. Data were obtained from triplicate wells per condition and were representative of at least three independent experiments.

\section{Western blot}

The total proteins of the cells were extracted using lysis buffer containing $1 \%$ protease inhibitor cocktail and phosphatase inhibitor cocktail for $30 \mathrm{~min}$ at $4^{\circ} \mathrm{C}$. Centrifugation was for $10 \mathrm{~min}$ at $4^{\circ} \mathrm{C}$, and the supernatants were used as the total protein samples. $10 \mu \mathrm{g}$ protein was separated using 10\% SDS-PAGE and transferred to polyvinylidene fluoride (PVDF) membranes. The PVDF membrane was incubated in $10 \%$ bovine serum albumin solution to block the nonspecific binding. SIX1 (1:1000, Sigma-Aldrich), GLUT1, GLUT2, GLUT3 (1:800, Abcam, Burlingame, CA, USA) and GAPDH (1:1000, Cell Signaling Technology, Danvers, MA, USA) antibodies were incubated at $4^{\circ} \mathrm{C}$ overnight. PVDF membranes were washed with TTBS buffer, and were incubated with horseradish peroxidase conjugated anti-mouse/rabbit IgG (1:1000, Cell Signaling Technology) for $2 \mathrm{~h}$ at $37^{\circ} \mathrm{C}$. Visualization was performed using ECL (Thermo Fisher, USA) and a DNR Biolmaging Systems (DNR, Israel).

\section{Glucose uptake}

Glucose uptake was determined using the 2-NBDG glucose uptake assay kit (Biovision, Milpitas, CA, USA). Assays were performed according to the manufacturer's instruction. Cells $\left(2-5 \times 10^{4}\right.$ cells/well $)$ were seeded 1 day before starting the assay. After 8-12 $h$, remove the culture medium (10\% FBS) was removed, and cells were incubated in $400 \mu \mathrm{L}$ cell culture medium with $0.5 \% \mathrm{FBS}$, at $37^{\circ} \mathrm{C}$ with $5 \% \mathrm{CO}_{2}$ for $1 \mathrm{~h}$. The cell culture medium was then removed, and $400 \mu \mathrm{L}$ of glucose uptake mix was added without disturbing the cells, and the cells were incubated at $37^{\circ} \mathrm{C}$ with $5 \% \mathrm{CO}_{2}$ for $30 \mathrm{~min}$. After incubation, the cells were harvested from the plate, kept on ice, washed once with $1 \mathrm{~mL}$ ice-cold $1 \times$ Analysis Buffer, centrifuged at $400 \times g$ for $5 \mathrm{~min}$, and the cell pellet was resuspended in $400 \mu \mathrm{L}$ of $1 \times$ Analysis Buffer, and analyzed by flow cytometry (488 $\mathrm{nm}$ excitation laser).

\section{ATP production assay}

ATP production was measured using an ATP assay kit (Abcam). The cells were lysed in $200 \mu \mathrm{L}$ of ATP Assay Buffer, centrifuged at 12,000 rpm for $5 \mathrm{~min}$ at $4^{\circ} \mathrm{C}$, and the protein was removed from the supernatant using a $10 \mathrm{kD}$ spin column (Thermo Scientific). $5 \mu \mathrm{L}$ of the de-proteinated sample was added to the ATP reaction mix in 96-well plates and a fluorescence reading at 535/587nm was measured.

\section{Luciferase reporter Assay}

FaDu cells were seeded into 24 well plates until the confluency reached $60 \%$. miR-23a-3p mimics, luciferase reporter and pRLTK vector carrying SIX1 3'-UTR (binding sites: AAUGUGAA) or SIX1 mutated 3'-UTR (binding sites: AAGGGCGA) were co-transfected into the cells. The transfected cells were cultured for another 24 hours in an incubator and then were lysed using $1 \times$ passive lysis buffer (Promega, San Luis Obispo, CA, USA) and the lysates were analyzed using the dual-luciferase reporter assay system (Promega). Independent experiments were performed in triplicate.

\section{Chromatin immunoprecipitation (ChIP)}

ChIP assay was performed using the Magna ChIP G Assay Kit (Millipore, Hayward, CA, USA) according to themanufacturer's instructions. Briefly, cellswere cross-linked with $37 \%$ formaldehyde, pelleted, and resuspended in lysis buffer. The cells were then sonicated and centrifuged to remove the insoluble material. The supernatants were collected and incubated overnight with indicated antibodies and Protein Gmagnetic beads. The beads were washed, and the precipitated chromatin complexes were collected, purified, and de-crosslinkedat $62^{\circ} \mathrm{C}$ for $2 \mathrm{~h}$, followed by incubation at $95^{\circ} \mathrm{C}$ for $10 \mathrm{~min}$. The precipitated DNA fragments were quantified using RT-PCR analysis. The primers for ChIP were as follows:

GLUT3 position1forward, 5' GGTGAATTGGAG AAAGTGTAT 3'; GLUT3 position1 reverse, 5' CCA CTCTTCTCTCTCCCCGAT3'; GLUT3 positions2 forward, $5^{\prime}$ AAGTATACCTACCCTTTGCCC3'; GLUT3 position 2reverse, 5' AAAACTATGAGGCA GGGAATG3'. 


\section{Statistical analysis}

Data was analyzed using SPSS 17.0 version for Windows (SPSS, Chicago, IL, USA). The possible correlations between SIX1 high levels and clinicopathological factors were determined using the Chi-Square test. Data between treated groups and control groups were determined using Student's t-test. A value of $p<0.05$ was considered to be statistically significant.

\section{Results}

\section{SIX 1 is overexpressed in HNSCC}

Immunohistochemistry of SIX1 was carried out in 102 cases of HNSCC specimens and 10 cases of normal tissues. Nuclear staining of SIX1 was identified as positive staining. Normal epithelial tissues exhibited negative or weak expression (Figure 1A,B), while positive nuclear SIX1 expression were found in $42.1 \%(43 / 102)$ of HNSCC tissues (Figure 1C-F).

We analyzed the correlation between SIX1 overexpression and the clinicopathological factors (Table 1). There was no difference between SIX1 status and age, sex and tumor differentiation. SIX1 overexpression positively correlated with advanced TNM stage $(p=0.0002)$, positive nodal metastasis (0.0297) and advanced T stage $(\mathrm{p}=0.021)$.

Table 1. Clinical profile and correlation between the clinicopathological features and SIX1 expression in HNSCC

\begin{tabular}{|c|c|c|c|c|}
\hline Characteristics & $\begin{array}{l}\text { Number of } \\
\text { patients }\end{array}$ & $\begin{array}{l}\text { SIX1 low } \\
\text { expression }\end{array}$ & $\begin{array}{l}\text { SIX1 high } \\
\text { expression }\end{array}$ & $P$ \\
\hline \multicolumn{5}{|l|}{ Age } \\
\hline$<60$ & 65 & 38 & 27 & 0.8669 \\
\hline$\geq 60$ & 37 & 21 & 16 & \\
\hline \multicolumn{5}{|l|}{ Gender } \\
\hline male & 72 & 39 & 33 & 0.2441 \\
\hline female & 30 & 20 & 10 & \\
\hline \multicolumn{5}{|l|}{ Differentiation } \\
\hline Well & 58 & 37 & 21 & 0.3081 \\
\hline Moderate & 28 & 13 & 15 & \\
\hline Poor & 16 & 9 & 7 & \\
\hline \multicolumn{5}{|l|}{ TNM stage } \\
\hline I+II & 70 & 49 & 21 & 0.0002 \\
\hline III+IV & 32 & 10 & 22 & \\
\hline \multicolumn{5}{|l|}{$\begin{array}{l}\text { Lymph node } \\
\text { metastasis }\end{array}$} \\
\hline Absent & 77 & 50 & 27 & 0.0297 \\
\hline Present & 25 & 9 & 16 & \\
\hline \multicolumn{5}{|l|}{ Tumor stage ( $\mathrm{T}$ ) } \\
\hline $\mathrm{T} 1+\mathrm{T} 2$ & 82 & 52 & 30 & 0.021 \\
\hline $\mathrm{T} 3+\mathrm{T} 4$ & 20 & 7 & 13 & \\
\hline
\end{tabular}

We also examined SIX1 expression in 10 pairs of HNSCC tissues with their adjacent normal tissues using western blots. We found that SIX1 protein expression was higher in 6 of 10 HNSCC tissues compared with their corresponding normal tissues (Figure 1G).
We then used the The Cancer Genome Atlas (TCGA) database for bioinformatics analyses. RNA-seq data from 496 cases of HNSCC with follow-up information were obtained from TCGA database. High SIX1 status was found to be associated with poor overall patient survival using the Kaplan-Meier plot $(p=0.0342$, log-rank test; Figure $1 \mathrm{H})$. We also performed Cox multivariate analyses using TCGA dataset. As shown in Supplementary Table 1, SIX1 was not an independent predicting factor for poor survival $(\mathrm{p}=0.0788)$. These results indicated that high SIX1 expression correlated with HNSCC progression.

\section{SIX1 regulates proliferation, ATP production, and glucose metabolism in HNSCC cells}

We profiled SIX1 protein expression in a panel of HNSCC cell lines (Figure 2A). The FaDu cell line had relatively low SIX1 expression while the Detroit562 and Eca-109 cell line had relatively high SIX1 expressions. We overexpressed SIX1 in FaDu cells and depleted SIX1 in Detroit562 cells. The transfection efficiency was confirmed by RT-qPCR and western blot (Figure 2B,C). Next the MTT and colony formation assays were conducted to test the effect of SIX1 on proliferation. The MTT assay results showed that the proliferation rate of Detroit562 cells decreased significantly after SIX1 depletion while SIX1 overexpression promoted proliferation rate of $\mathrm{FaDu}$ cells (Figure 2D). The colony formation assay results demonstrated that SIX1 positively regulated colony formation ability (Figure 2E).

Glucose uptake is the key step during glucose metabolism and ATP production. We measured glucose uptake using the 2-NBDG incorporation assay and found that SIX1 overexpression significantly increased the glucose uptake rate in FaDu cells while SIX1 depletion decreased the glucose uptake in Detroit562 cells (Figure 2F).These results indicated that SIX1 was as a key regulator of glucose uptake in HNSCC cells.

Cancer cells, including HNSCC, usually adopt aerobic glycolysis of glucose to produce energy (ATP), which is essential for cancer cell survival and malignant growth. To determine if SIX1 regulated metabolism in HNSCC cells, we examined the change of ATP production. As shown in Figure 2G, SIX1 overexpression increased ATP production in $\mathrm{FaDu}$ cells while SIX1 depletion decreased ATP production in Detroit562 cells. These data indicated that SIX1 enhanced the rate of metabolism in HNSCC cells.

According to a previous publication, an esophageal cancer cell line Eca-109 have high endogenous SIX1 expression [32]. We used it as a 
positive control cell line and performed siRNA knock down and biological experiments. As shown in Supplementary Figure 1, knockdown of SIX1 effectively downregulated cell growth, glucose

A

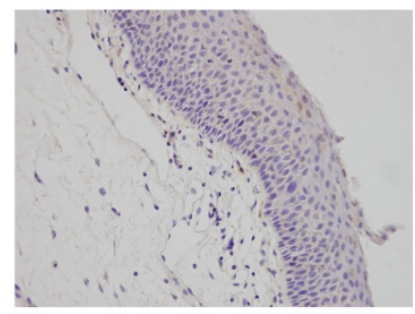

C

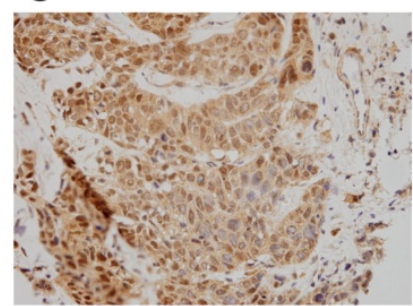

E

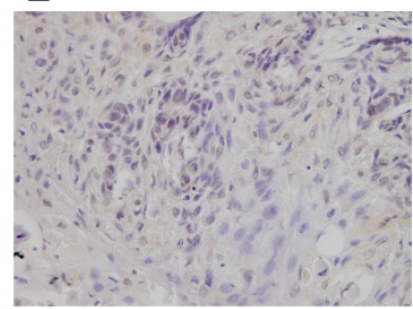

uptake and ATP production, suggesting the effect of SIX1 on glucose metabolism was also observed on other types of cancers.

\section{B}

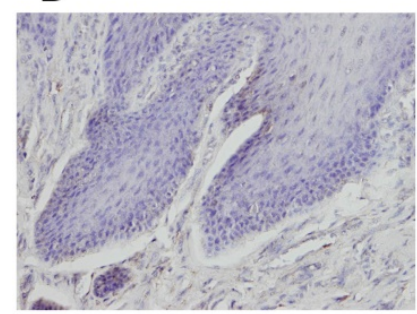

D

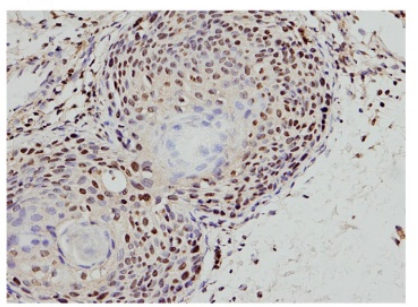

$\mathrm{F}$

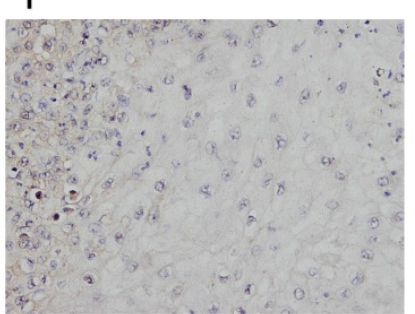

G

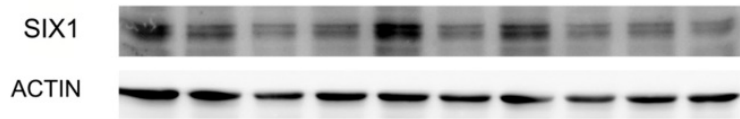

$\begin{array}{llllllllllll}1 \mathrm{~T} & 1 \mathrm{~N} & 2 \mathrm{~T} & 2 \mathrm{~N} & 3 \mathrm{~T} & 3 \mathrm{~N} & 4 \mathrm{~T} & 4 \mathrm{~N} & 5 \mathrm{~T} & 5 \mathrm{~N}\end{array}$
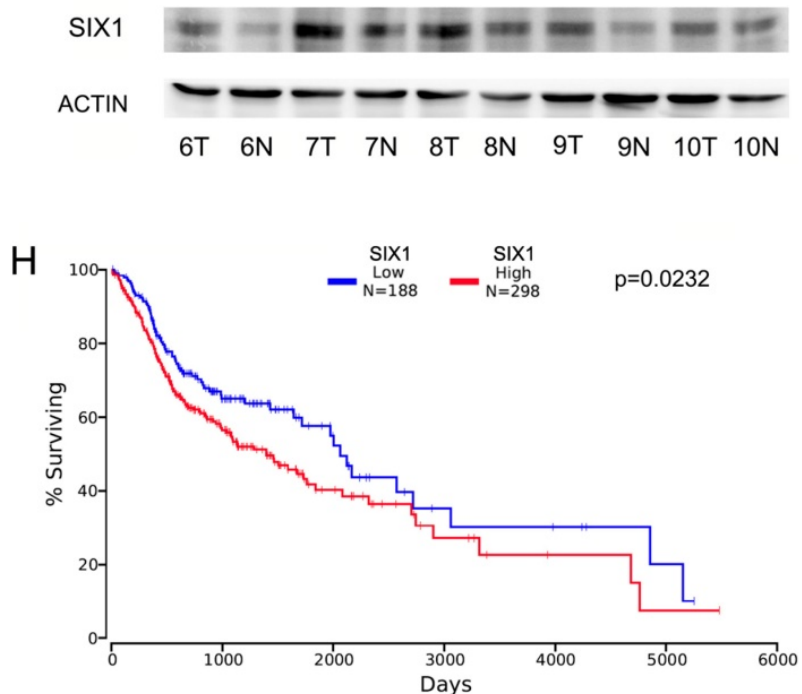

Figure 1. Expression of SIXI in HNSCC. A. Negative SIX1 expression in normal laryngeal squamous epithelium. B. Negative SIX1 expression in a case of normal oral epithelial tissue. C. Positive nuclear and cytoplasmic SIX1 expression in a case of laryngeal squamous cell carcinoma. D. Positive nuclear SIX1 expression a case of oral squamous cell carcinoma. E. Negative SIXI expression in a case of laryngeal squamous cell carcinoma. F. Negative SIX1 expression in a case of oral squamous cell carcinoma. G. Western blot analysis of SIX1 protein expression in 10 cases of HNSCC tissues and their adjacent normal tissues. H. External datasets from TCGA showed that overexpression of SIX 1 correlated with poor overall survival of HNSCC. 
A

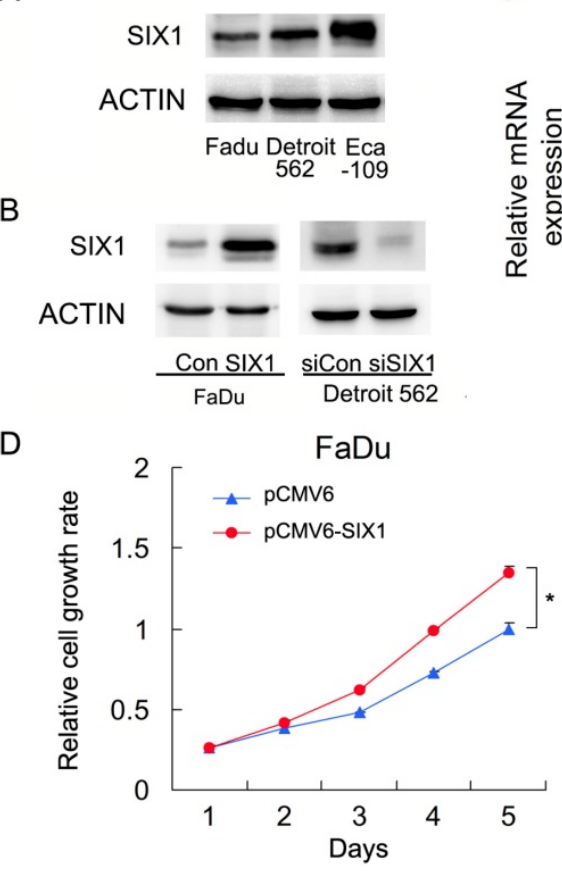

E
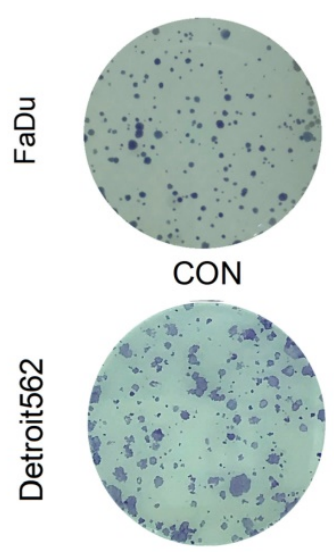

F

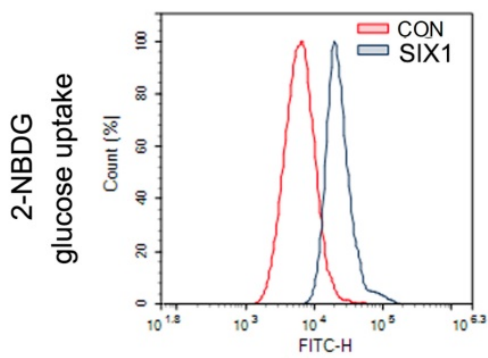

G
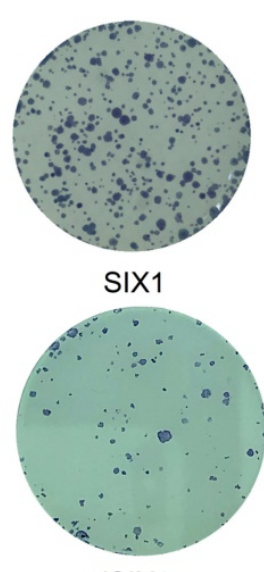

sisIX1

C

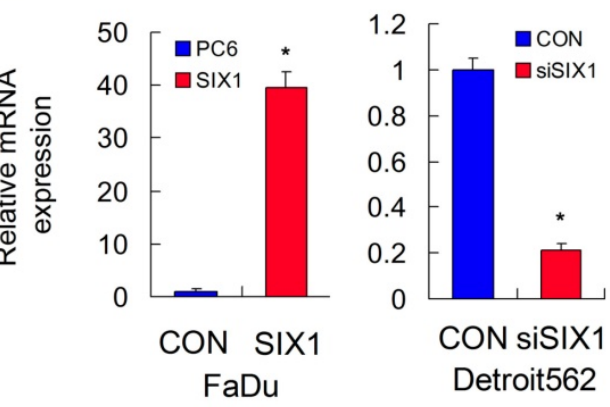

Detroit562
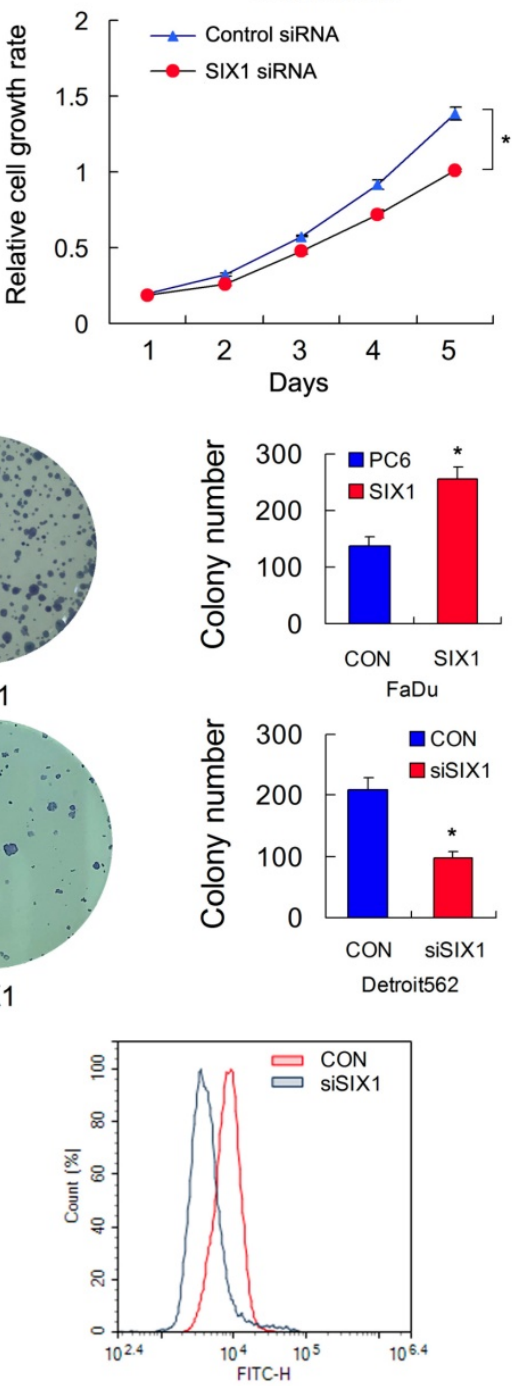

Detroit562
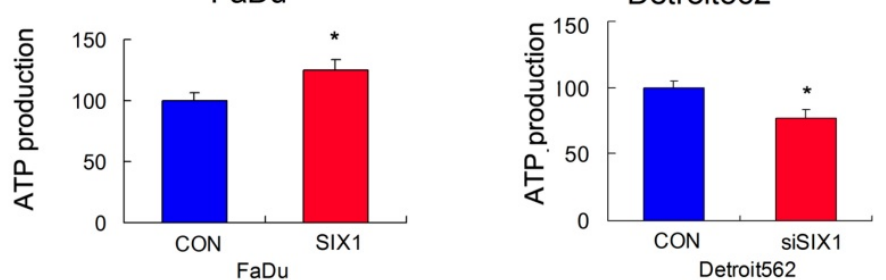

Figure 2. SIX1 regulates glucose metabolism and ATP production in HNSCC cells. A. SIX1 protein expression in a $4 \mathrm{HNSCC}$ cell lines. B and C. Efficiencies of SIX 1 plasmid transfection in FaDu cell line siRNA knockdown in Detroit562 cell line. D. MTT assay showed that SIX1 overexpression promoted proliferation rate in FaDu cell line. SIX1 depletion inhibited proliferation rate in Detroit562 cell line. E. Colony formation assay demonstrated that SIX1 overexpression upregulated colony number in FaDu cell line while SIX1 depletion downregulated colony number in Detroit562 cell line. F. 2-NBDG glucose uptake assay demonstrated that SIX1 overexpression upregulated glucose uptake in FaDu cell line. SIX1 depletion showed the opposite effect in Detroit562 cell line. G. SIX1 overexpression increased ATP production in FaDu cells while SIX1 depletion decreased ATP production in Detroit562 cells. * indicates $p<0.05$. 


\section{SIX1 regulates GLUT3 expression in HNSCC}

To identify the mechanism of SIX1 on glucose uptake, we screened genes related to glucose metabolism using RT-qPCR and western blots. We found that SIX1 overexpression increased protein and mRNA expression of GLUT3 in FaDu cells. In contrast, SIX1 downregulation suppressed protein and mRNA expression of GLUT3 (Figure 3A, B). Because SIX1 has been reported as a transcription regulator, we investigated if SIX1 regulated GLUT3 by binding to its promoter. We used TRANSFAT software to predict the potential binding sites of SIX1 on the GLUT3 promoter (Figure 3C). Binding of SIX1 to the GLUT3 promoter region was validated using chromatin immunoprecipitation (ChIP) assay with anti-SIX1 antibody followed by RT-QPCR assay in FaDu cells (Figure 3D). These results indicated that SIX1 promoted glucose uptake by transcriptional regulation of GLUT3 in HNSCCs.

\section{4. miR-23a-3p targets and downregulates $S|X|$ in HNSCC cells}

Malignant cells show dependence on dysregulated miRNAs, which in turn control protein coding oncogenes. To identify potential miRNAs that could downregulate SIX1, we used three target prediction software (miRanda, miRwalk and
TargetScan). Among the common miRNAs shared by the results from these 3 programs, we mainly focused on miR-23a-3p. We showed that the miR-23a-3p mimic repressed SIX1 expression both at the mRNA and protein levels in $\mathrm{FaDu}$ cells. In contrast, the miR-23a-3p inhibitor upregulated SIX1 mRNA and protein expressions (Figure 4 A,B). The putative binding site of miR-23a-3p to the 3'-UTR of SIX1, which was predicted using Targetscan, is shown in Figure $4 \mathrm{C}$. To validate the regulation of miR-23a-3p on SIX1, luciferase reporter assays were performed. The ratio of fluorescence intensity for wild-type and the mutant binding sites was calculated. The miR-23a-3p mimic reduced the luciferase intensity in $\mathrm{FaDu}$ cells transfected with vector containing wild-type 3'-UTR, while no significant change was observed in $\mathrm{FaDu}$ cells transfected with vector containing mutant binding site, indicating that miR-23a-3p bound to the SIX1 3'-UTR region directly (Figure 4D). To further validate the relationship between miR-23a-3p and SIX1 in HNSCC tissues, we analyzed TCGA data and correlated miR-23a-3p with SIX1 mRNA using linear regression. As shown in Figure 4E, there was a significantly negative correlation between miR-23a-3p and SIX1 expression using linear regression, which further supported our results that miR-23a-3p downregulated SIX1 in HNSCC.
A

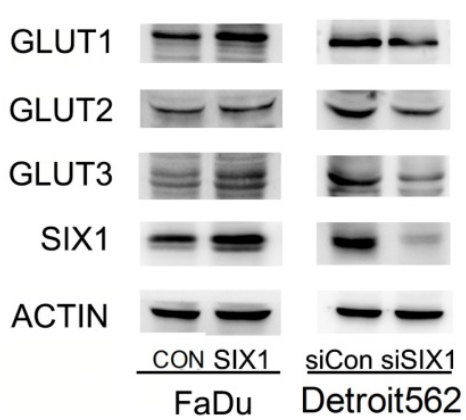

C

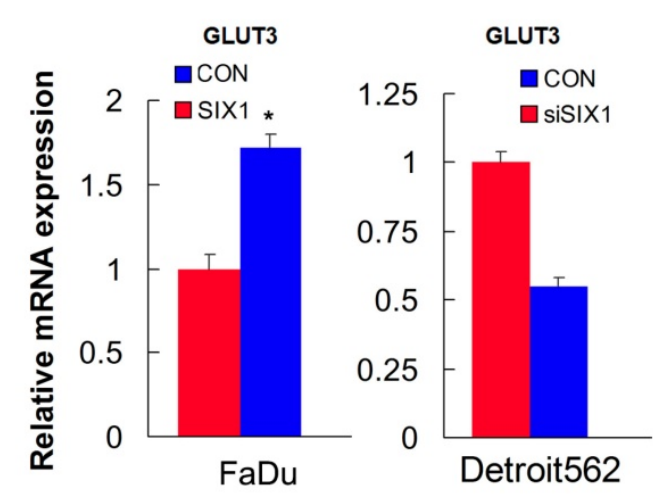

D

SIX1 binding position on GLUT3 promoter
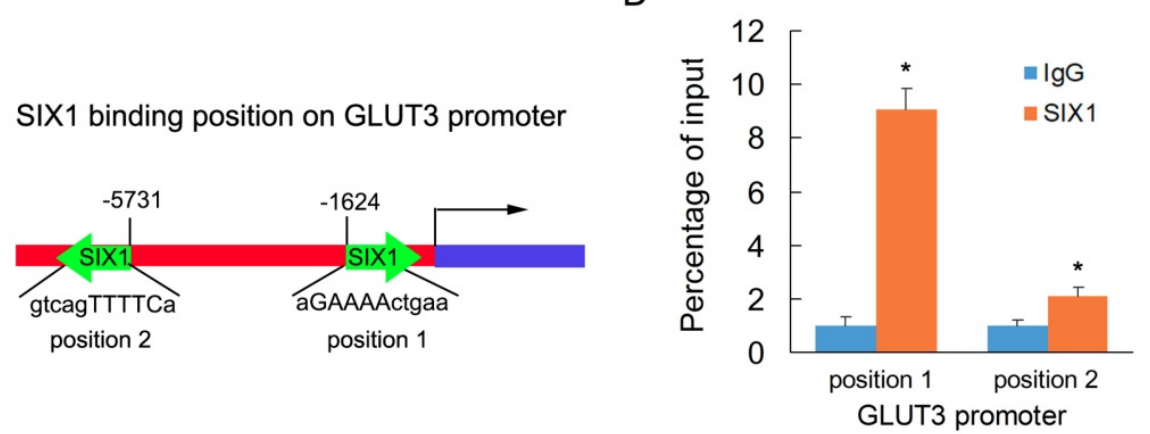

Figure 3. SIX1 regulates GLUT3 in HNSCC. A. Western blot showed regulation of SIX1 on GLUT family proteins. B. Real-time PCR shows the effects of SIX1 on GLUT3. C. Prediction of binding sites and sequences by TRANSFAC database analysis. D. ChIP assay showed that SIX1could bind to the GLUT3 promoter. $*$ indicates $P<0.05$. 
A

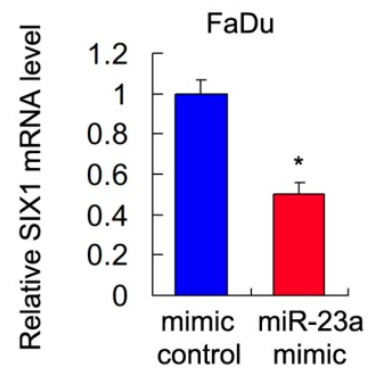

B

SIX1

ACTIN

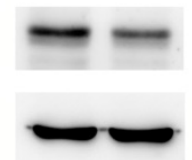

mimic miR-23a

control mimic
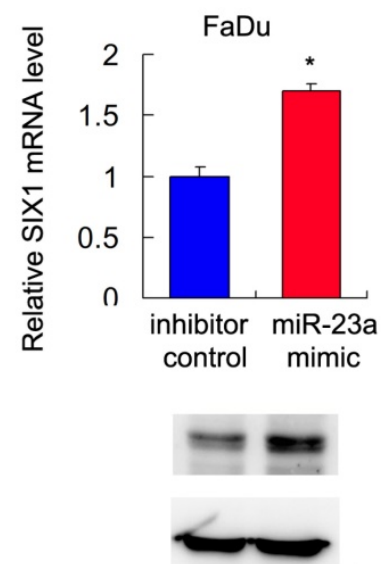

inhibitor miR-23a

control mimic

C

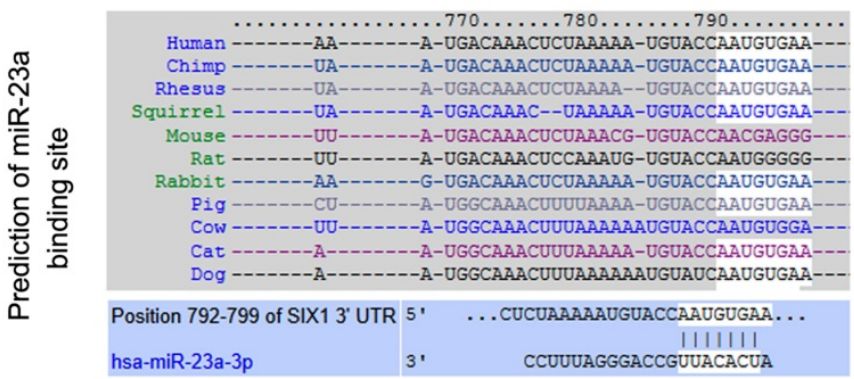

D

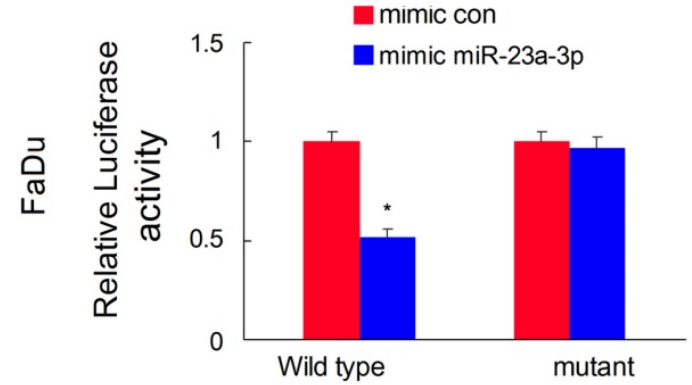

E

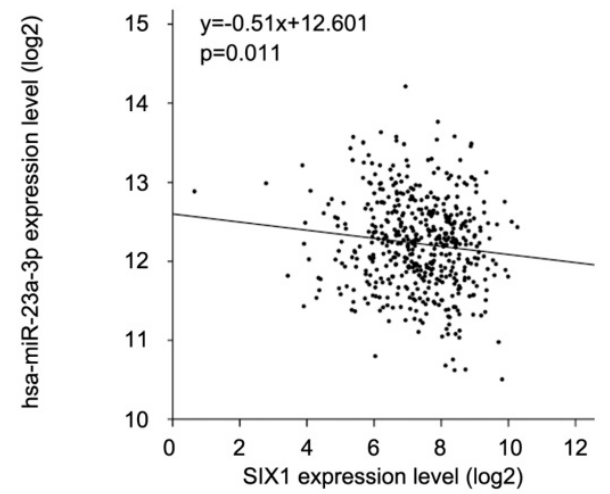

Figure 4. miR-23a-3p targets and downregulates SIX1 in HNSCC. A and B. miR-23-3p regulates SIX1 mRNA and protein expression in FaDu cells. C. Prediction of SIX1 as a target of miR-23a-3p using Targetscan analysis. D. miR-23a-3p mimic suppressed the luciferase reporter activity of wild-type reporter, while no significant change was observed in that of mutant reporter. E. TCGA data showed that there was a significant negative correlation between miR-23a and SIX1 expression using linear regression. * indicates $\mathrm{p}<0.05$.

\section{5. miR-23a-3p regulates GLUT3 and glucose uptake via SIXI in HNSCC cells}

To investigate if miR-23a-3p regulated glucose metabolism in HNSCC cells, we transfected FaDu cells with the miR-23a-3p mimic, with or without SIX1 plasmid. We then examined glucose uptake and GLUT3 expression. As shown in Figure 5A and B, the miR-23a mimic downregulated GLUT3 protein expression. SIX1 partly restored GLUT3 expression in 
A

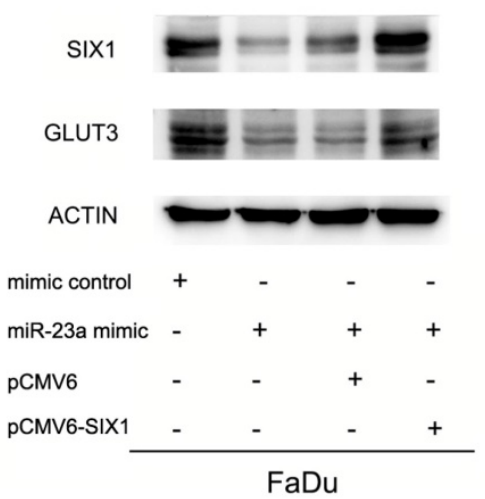

C

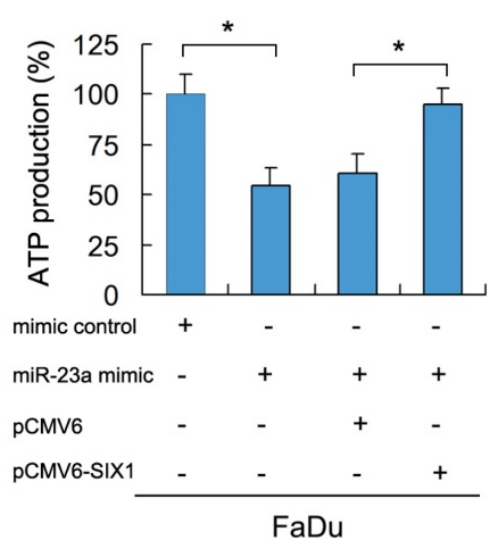

B
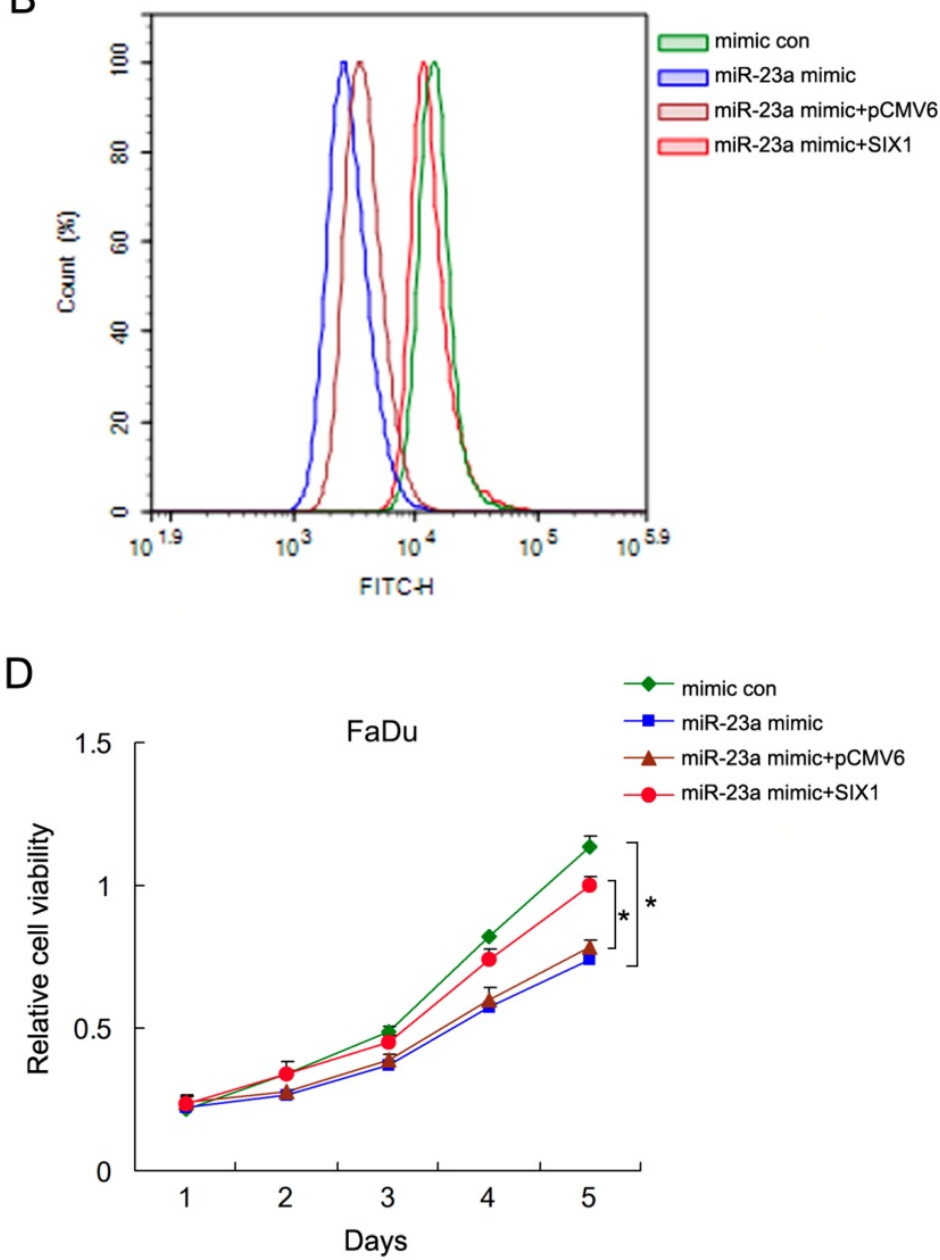

Figure 5. miR-23a-3p regulates glucose uptake via SIXI in HNSCC cells. A. Western blot demonstrated that miR-23a-3p mimic suppressed GLUT3 protein, which was partly restored by SIX1 in FaDu cells. B and C. 2NBDG glucose uptake and ATP production assays showed that miR-23a-3p mimic suppressed glucose uptake, which could be restored by SIX1 overexpression. D. MTT assay showed that miR-23a-3p decreased proliferation of FaDu cells, which was restored by SIXI overexpression. * indicates $\mathrm{p}<0.05$.

cells transfected with the miR-23a-3p mimic (Figure 5A). The 2-NBDG glucose uptake assay results demonstrated that the miR-23a-3p mimic decreased glucose uptake, which could be restored by SIX1 plasmid transfection (Figure 5B). Analysis of ATP levels also showed that the miR-23a-3p mimic inhibited ATP production, which was rescued by SIX1 overexpression (Figure 5C). MTT assay showed that miR-23a-3p decreased FaDu cell growth, which was restored by SIX1 transfection (Figure 5D). These results indicated that miR-23a-3p regulated glucose metabolism and ATP production by targeting and downregulating SIX1 in HNSCC cells.

\section{Discussion}

Recently, evidence has shown that SIX1 is overexpressed in various cancers including breast, ovarian, colorectal and liver cancer [10-12, 15-17]. However, the clinical significance of SIX1 in HNSCC remains unexplored. To address this issue, we checked protein expression patterns of SIX1 and found that SIX1 was overexpressed in HNSCC tissues. High SIX1 status positively correlated with advanced TNM stage and the presence of lymph node metastasis. Importantly, analysis of TCGA data demonstrated that SIX1 overexpression correlated with poor patient survival, making SIX1 a potential prognostic cancer marker in HNSCC.

To explore its biological roles, we examined the role of SIX1 on cell proliferation using the MTT and colony formation assays. Our data demonstrated that SIX1 overexpression promoted cell growth and colony formation ability, while SIX1 depletion showed the opposite effects. Tumor cells tend to shift their metabolism toward aerobic glycolysis, thus producing ATP. Glucose transport is the initial step during glucose metabolism in tumor cells. Here, we found that SIX1 overexpression facilitated glucose uptake into tumor cells and increased ATP 
production in HNSCC cells, suggesting that SIX1 was a positive regulator of glucose metabolism in HNSCC.

Glucose transporter proteins form membrane transporters that mediate the transport of small carbon compounds across the membranes. GLUT proteins are encoded by the SLC2 genes and are members of the major facilitator superfamily of membrane transporter proteins. In accordance with the high glucose consumption, the GLUT family has been found to be overexpressed in various cancers [33-35]. Here, we screened several GLUT family proteins and found that SIX1 mainly upregulated GLUT3 expression at both mRNA and protein levels. According to the prediction of TRANSFAC software, GLUT3 possessed 2 regulatory sites for SIX1, which was confirmed using the ChIP assay. Our results clearly demonstrated that SIX1 was a critical positive regulator of GLUT3 in HNSCC cells. GLUT3 is a highly active glucose transporter, which has been reported as a potential target for anticancer therapy $[36,37]$. In addition, GLUT3 is overexpressed in HNSCC, which serves as an indicator of poor prognosis [38]. Our findings elucidated a novel pathway for GLUT3 upregulation in HNSCC and suggested that a GLUT3 targeting strategy could be achieved by targeting SIX1.

Malignant cells show dependence on the dysregulated miRNAs, which in turn control protein coding oncogenes. These miRNAs provide important opportunities for development of future miRNA-based therapies. Using prediction software, we found that SIX1 was a potential regulator of miR-23a-3p. The biological role of miR-23a-3p has been controversial. Upregulation of miR-23a-3p induced caspase-dependent and -independent apoptosis [39]. Overexpression of miR-23a-3p could induced cancer cell hypersensitivity to chemotherapy in vitro and in vivo [40]. In addition, miR-23a-3p has been reported to negatively regulate ATP production and metabolism by targeting mitochondrial glutaminase [41]. These reports suggested that miR-23a-3p was a negative regulator of cancer growth and metabolism. In the current study, we confirmed that miR-23a-3p targeted and downregulated SIX1 using RT-qPCR, western blots and the luciferase reporter assay. This was further supported by the analysis of TCGA data showing a negative relationship between SIX1 and miR-23a expression. In addition, our results demonstrated that miR-23a-3p downregulated GLUT3 expression, inhibited glucose uptake, ATP production and HNSCC cell proliferation, which could be rescued by restoration of SIX1 expression. Our data confirmed that miR-23a served as a negative regulator of glucose uptake and metabolism by targeting SIX1/GLUT3 signaling in HNSCC.

In conclusion, our data demonstrated that SIX1 was overexpressed in HNSCC and promoted cancer progression and glucose uptake by transcriptional upregulation of GLUT3. miR-23a downregulated GLUT3 and glucose uptake by targeting SIX1 in HNSCC.

\section{Supplementary Material}

Supplementary figure and table. http://www.jcancer.org/v11p2529s1.pdf

\section{Acknowledgements}

The study was supported by The Natural Science Foundation of Liaoning (No. 20180540097).

\section{Competing Interests}

The authors have declared that no competing interest exists.

\section{References}

1. Agrawal N, Frederick MJ, Pickering CR, Bettegowda C, Chang K, Li RJ, et al. Exome sequencing of head and neck squamous cell carcinoma reveals inactivating mutations in NOTCH1. Science. 2011; 333: 1154-7.

2. Leemans CR, Braakhuis BJ, Brakenhoff RH. The molecular biology of head and neck cancer. Nat Rev Cancer. 2011; 11: 9-22.

3. Chow LQM, Haddad R, Gupta S, Mahipal A, Mehra R, Tahara M, et al. Antitumor Activity of Pembrolizumab in Biomarker-Unselected Patients With Recurrent and/or Metastatic Head and Neck Squamous Cell Carcinoma: Results From the Phase Ib KEYNOTE-012 Expansion Cohort. J Clin Oncol. 2016; 34: 3838-45.

4. Kumar JP. The sine oculis homeobox (SIX) family of transcription factors as regulators of development and disease. Cell Mol Life Sci. 2009; 66: 565-83.

5. Lu J, Getz G, Miska EA, Alvarez-Saavedra E, Lamb J, Peck D, et al. MicroRNA expression profiles classify human cancers. Nature. 2005; 435: 834-8.

6. Tavares ALP, Cox TC, Maxson RM, Ford HL, Clouthier DE. Negative regulation of endothelin signaling by SIX1 is required for proper maxillary development. Development. 2017; 144: 2021-31.

7. Fujimoto Y, Tanaka SS, Yamaguchi YL, Kobayashi H, Kuroki S, Tachibana M, et al. Homeoproteins Six1 and Six4 regulate male sex determination and mouse gonadal development. Dev Cell. 2013; 26: 416-30.

8. Li X, Oghi KA, Zhang J, Krones A, Bush KT, Glass CK, et al. Eya protein phosphatase activity regulates Six1-Dach-Eya transcriptional effects in mammalian organogenesis. Nature. 2003; 426: 247-54.

9. Zhang $\mathrm{H}$, Stavnezer E. Ski regulates muscle terminal differentiation by transcriptional activation of Myog in a complex with Six1 and Eya3. J Biol Chem. 2009; 284: 2867-79.

10. Smith AL, Iwanaga R, Drasin DJ, Micalizzi DS, Vartuli RL, Tan AC, et al. The miR-106b-25 cluster targets Smad7, activates TGF-beta signaling, and induces EMT and tumor initiating cell characteristics downstream of Six1 in human breast cancer. Oncogene. 2012; 31: 5162-71.

11. Wang CA, Jedlicka P, Patrick AN, Micalizzi DS, Lemmer KC, Deitsch E, et al. SIX1 induces lymphangiogenesis and metastasis via upregulation of VEGF-C in mouse models of breast cancer. J Clin Invest. 2012; 122: 1895-906.

12. Behbakht K, Qamar L, Aldridge CS, Coletta RD, Davidson SA, Thorburn A, et al. Six1 overexpression in ovarian carcinoma causes resistance to TRAIL-mediated apoptosis and is associated with poor survival. Cancer Res. 2007; 67: 3036-42.

13. Wegert J, Ishaque N, Vardapour R, Georg C, Gu Z, Bieg M, et al. Mutations in the SIX1/2 pathway and the DROSHA/DGCR8 miRNA microprocessor complex underlie high-risk blastemal type Wilms tumors. Cancer Cell. 2015; 27: $298-311$

14. Mimae T, Okada M, Hagiyama M, Miyata $Y$, Tsutani $\mathrm{Y}$, Inoue $\mathrm{T}$, et al. Upregulation of notch2 and six1 is associated with progression of early-stage lung adenocarcinoma and a more aggressive phenotype at advanced stages. Clin Cancer Res. 2012; 18: 945-55.

15. Ng KT, Man K, Sun CK, Lee TK, Poon RT, Lo CM, et al. Clinicopathological significance of homeoprotein Six1 in hepatocellular carcinoma. British journal of cancer. 2006; 95: 1050-5. 
16. Ng KT, Lee TK, Cheng Q, Wo JY, Sun CK, Guo DY, et al. Suppression of tumorigenesis and metastasis of hepatocellular carcinoma by shRNA interference targeting on homeoprotein Six1. Int J Cancer. 2010; 127: 859-72.

17. Ono H, Imoto I, Kozaki K, Tsuda H, Matsui T, Kurasawa $\mathrm{Y}$, et al. SIX1 promotes epithelial-mesenchymal transition in colorectal cancer through ZEB1 activation. Oncogene. 2012; 31: 4923-34.

18. Riddiford N, Schlosser G. Six1 and Eya1 both promote and arrest neuronal differentiation by activating multiple Notch pathway genes. Dev Biol. 2017; 431: 152-67.

19. Li Z, Tian T, Lv F, Chang Y, Wang X, Zhang L, et al. Six1 promotes proliferation of pancreatic cancer cells via upregulation of cyclin D1 expression. PLoS One. 2013; 8: e59203.

20. Tian T, Li A, Lu H, Luo R, Zhang M, Li Z. Six1 promotes glioblastoma cell proliferation and invasion by upregulation of connective tissue growth factor. Am J Cancer Res. 2015; 5: 1823-30.

21. Radisky DC. Defining a role for the homeoprotein Six1 in EMT and mammary tumorigenesis. J Clin Invest. 2009; 119: 2528-31.

22. Coletta RD, Christensen KL, Micalizzi DS, Jedlicka P, Varella-Garcia M, Ford HL. Six1 overexpression in mammary cells induces genomic instability and is sufficient for malignant transformation. Cancer Res. 2008; 68: 2204-13.

23. Zhang $\mathrm{X}, \mathrm{Xu} \mathrm{R}$. Six 1 expression is associated with a poor prognosis in patients with glioma. Oncol Lett. 2017; 13: 1293-8.

24. Iwanaga R, Wang CA, Micalizzi DS, Harrell JC, Jedlicka P, Sartorius CA, et al. Expression of Six1 in luminal breast cancers predicts poor prognosis and promotes increases in tumor initiating cells by activation of extracellular signal-regulated kinase and transforming growth factor-beta signaling pathways. Breast Cancer Res. 2012; 14: R100.

25. Chao L, Liu J, Zhao D. Increased Six1 expression is associated with poor prognosis in patients with osteosarcoma. Oncol Lett. 2017; 13: 2891-6.

26. Rupaimoole R, Slack FJ. MicroRNA therapeutics: towards a new era for the management of cancer and other diseases. Nat Rev Drug Discov. 2017; 16: 203-22

27. Friedman RC, Farh KK, Burge CB, Bartel DP. Most mammalian mRNAs are conserved targets of microRNAs. Genome Res. 2009; 19: 92-105.

28. Volinia S, Calin GA, Liu CG, Ambs S, Cimmino A, Petrocca F, et al. A microRNA expression signature of human solid tumors defines cancer gene targets. Proc Natl Acad Sci U S A. 2006; 103: 2257-61.

29. Torre LA, Bray F, Siegel RL, Ferlay J, Lortet-Tieulent J, Jemal A. Global cancer statistics, 2012. CA Cancer J Clin. 2015; 65: 87-108.

30. Kinoshita T, Nohata N, Hanazawa T, Kikkawa N, Yamamoto N, Yoshino H, et al. Tumour-suppressive microRNA-29s inhibit cancer cell migration and invasion by targeting laminin-integrin signalling in head and neck squamous cell carcinoma. British journal of cancer. 2013; 109: 2636-45.

31. Hersi HM, Raulf N, Gaken J, Folarin N, Tavassoli M. MicroRNA-9 inhibits growth and invasion of head and neck cancer cells and is a predictive biomarker of response to plerixafor, an inhibitor of its target CXCR4. Molecular oncology. 2018; 12: 2023-41.

32. He Z, Li G, Tang L, Li Y. SIX1 overexpression predicts poor prognosis and induces radioresistance through AKT signaling in esophageal squamous cell carcinoma. Onco Targets Ther. 2017; 10: 1071-9.

33. Pizzuti L, Sergi D, Mandoj C, Antoniani B, Sperati F, Chirico A, et al. GLUT 1 receptor expression and circulating levels of fasting glucose in high grade serous ovarian cancer. J Cell Physiol. 2018; 233: 1396-401.

34. Chai YJ, Yi JW, Oh SW, Kim YA, Yi KH, Kim JH, et al. Upregulation of SLC2 (GLUT) family genes is related to poor survival outcomes in papillary thyroid carcinoma: Analysis of data from The Cancer Genome Atlas. Surgery. 2017; 161: $188-94$

35. Cho H, Lee YS, Kim J, Chung JY, Kim JH. Overexpression of glucose transporter-1 (GLUT-1) predicts poor prognosis in epithelial ovarian cancer. Cancer Invest. 2013; 31: 607-15.

36. Masin M, Vazquez J, Rossi S, Groeneveld S, Samson N, Schwalie PC, et al. GLUT3 is induced during epithelial-mesenchymal transition and promotes tumor cell proliferation in non-small cell lung cancer. Cancer Metab. 2014; 2: 11.

37. Meneses AM, Medina RA, Kato S, Pinto M, Jaque MP, Lizama I, et al. Regulation of GLUT3 and glucose uptake by the cAMP signalling pathway in the breast cancer cell line ZR-75. J Cell Physiol. 2008; 214: 110-6.

38. Ayala FR, Rocha RM, Carvalho KC, Carvalho AL, da Cunha IW, Lourenco SV, et al. GLUT1 and GLUT3 as potential prognostic markers for Oral Squamous Cell Carcinoma. Molecules. 2010; 15: 2374-87.

39. Chhabra R, Adlakha YK, Hariharan M, Scaria V, Saini N. Upregulation of miR-23a-27a-24-2 cluster induces caspase-dependent and -independent apoptosis in human embryonic kidney cells. PLoS One. 2009; 4: e5848.

40. Wang N, Zhu M, Tsao SW, Man K, Zhang Z, Feng Y. MiR-23a-mediated inhibition of topoisomerase 1 expression potentiates cell response to etoposide in human hepatocellular carcinoma. Mol Cancer. 2013; 12: 119.

41. Gao P, Tchernyshyov I, Chang TC, Lee YS, Kita K, Ochi T, et al. c-Myc suppression of miR-23a/b enhances mitochondrial glutaminase expression and glutamine metabolism. Nature. 2009; 458: 762-5. 